\title{
Femtosecond Laser-Assisted Cataract Surgery in Soft and Hard Nuclear Cataracts: A Comparison of Effective Phacoemulsification Time
}

This article was published in the following Dove Press journal: Clinical Ophthalmology

\author{
Ahmed H Assaf ${ }^{1,2}$ \\ Mohamed G Aly iD ' \\ Rania G Zaki ${ }^{1}$ \\ Yasmine M Shaaban (D) \\ Bassem F Aziz (iD) ${ }^{1,2}$ \\ 'Ain Shams University, Faculty of \\ Medicine, Ophthalmology Department, \\ Cairo, Egypt; ${ }^{2}$ Al Watany Eye Hospital, \\ Cataract and Refractive Department, \\ Cairo, Egypt
}

Purpose: To compare effective phacoemulsification time (EFX) in femtosecond laserassisted cataract surgery (FLACS) versus traditional quick chop phacoemulsification (QCP) in senile nuclear cataracts with different densities focusing on soft and hard ones.

Patients and Methods: A prospective non-randomized comparative study was carried out in Al Watany Eye Hospital and Ain Shams University Hospital, Cairo, Egypt; 250 eyes with senile nuclear cataract (NC) were included and classified into two main groups, FLACS and QCP groups. Each of them was stratified according to nuclear density into three subgroups, subgroups I (Soft NC), II (Medium NC), and III (Hard NC). Sextans-softened fragmentation pattern was performed in the FLACS group. Total EFX utilized for nucleus disassembly and removal was recorded by the completion of each surgery.

Results: A total of 117 eyes were included in the FLACS group and 133 eyes in the QCP group. No significant difference in EFX was observed between the two groups $(P=0.228)$. Regarding subgroups, EFX showed no statistically significant difference between FLACS and QCP $(P=0.283)$ in soft NC. For hard NC, a trend to lower values of EFX in FLACS compared with QCP was found, but without statistically significant difference $(P=0.122)$. Only in medium NC were significantly lower values obtained in FLACS compared with QCP $(P<0.0001)$.

Conclusion: When compared with QCP technique, FLACS can be used for advantages aside from EFX reduction, including astigmatic keratotomies, accurate sizing, and centration of capsulotomies especially in hard and soft nuclear cataracts. Significant reduction of total EFX with FLACS is most prominent only in medium-density nuclear cataracts.

Keywords: phacoemulsification, EFX, FLACS, quick chop, femtosecond laser, cataract surgery

\section{Introduction}

Femtosecond laser (FL) usage has become increasingly popular in phacoemulsification surgery specifically in doing corneal incisions, anterior capsulorhexis, and lens fragmentation. ${ }^{1}$ Several researches have stated excellent clinical outcomes with this technology ${ }^{2-4}$ with some potential advantages over conventional phacoemulsification (CP). ${ }^{5}$ In particular, femtosecond laser-assisted cataract surgery (FLACS) is nowadays advertised as being superior over CP regarding effective phacoemulsification time, ${ }^{6-8}$ preservation of corneal endothelial integrity, reduction of postoperative corneal edema, ${ }^{6,9,10}$ decreasing the risk of intraoperative vitreous loss, ${ }^{11}$ and in management of complex cases. ${ }^{12}$ In contrast, no clear benefit of FLACS over CP has been found in terms
Correspondence: Ahmed H Assaf Ain Shams University, Cairo, Egypt Email assaf.ahmed@gmail.com 
of visual outcomes or complications. ${ }^{13-17}$ On the other hand, some studies have reported the absence of significant differences between the two techniques regarding phacoemulsification time. ${ }^{13}$ This variability between comparative studies of FLACS versus CP might be attributed to the inclusion of cataracts with different nuclear densities with subsequent different surgical requirements, and the used technique of $\mathrm{CP}$ whether divide-and-conquer, stop-and-chop, or quick-chop.

Regarding nuclear densities, many research and metaanalysis studies focused on medium density cataract and found significant differences between FLACS and CP as regards efficacy and safety. Yet, difficult hard nuclear cataracts were not included in most of the published studies in their analysis. Thus, the hard nuclear cataract is considered a point of further investigation. ${ }^{18,19}$

Thus, the purpose of the current study was to assess the total measured effective phacoemulsification time (EFX) used in FLACS compared to traditional quick chop phacoemulsification (QCP) in relation to different nuclear densities with special focus on soft and hard nuclear cataract. We focused only on energy consumption between FLACS and QCP, and we did not compare the effects of both procedures on the surrounding tissues like corneal endothelium.

\section{Patients and Methods}

This prospective non-randomized comparative study was conducted at Al Watany Eye hospital and Ain Shams University Hospital, Cairo, Egypt, within a time interval from March 2018 to March 2019. The study is adherent to the tenets of the Declaration of Helsinki and gained approval by the ethical committee of Watany Research and Development Center in Al Watany Eye Hospital, and informed consents for the research were obtained from all patients.

Eyes with senile nuclear cataract with corrected distance visual acuity worse than +0.3 on the Log MAR scale were selected. All patients set up for cataract surgery were adequately informed about FLACS and QCP including advantages, cost, and disadvantages. Both procedures were explained to all participants and given to them as reliable options by medical staff. The patient's decision was according to his/her preference. All participants signed a consent form and agreed to be enrolled in our study.

Both FLACS and QCP groups were sub-classified into three subgroups according to density of nuclear cataract. The nuclear cataract (NC) color was considered on the scale of 1 to 6 for all eyes by an ophthalmologist (BFA) who used an SC5 slit lamp (Topcon, Japan) to determine the NC color depending on the Lens Opacities Classification System III (LOCS III) ${ }^{20}$ which depends on cataract color in grading and is considered a subjective method. Then we confirmed the density classification by using the Pentacam Nucleus Staging (PNS) function, which is an objective function incorporated in the Pentacam software (OCULUS, Wetzlar, Germany), depending only on the Pentacam machine. We used both methods and matched them together and classified the cases into three subgroups; subgroup I comprised eyes with soft NC 1-2; subgroup II, medium NC 3-4; and subgroup III, hard NC 5-6.

We calculated the minimal sample size required in FLACS and QCP by conservative G*Power software version 3.1.9.2, Type I error (Alpha, 0.05) and Type II error (Beta, 0.80). With reference to two original articles, Hida et al and Aly et al, ${ }^{21,22}$ we found that the minimum sample size of the FLACS group must not be less than 40 (in our paper $=117$ ) and in QCP must not be less than 40 (in our paper $=133)$.

We excluded eyes with glaucoma, weak zonules, subluxated lenses, corneal opacities, maximum pupillary dilation of less than $5.50 \mathrm{~mm}$, history of uveitis, abnormal iris, and intraoperative complications.

\section{Surgical Techniques}

The same experienced surgeon (AHA) performed all the surgeries in FLACS and QCP under topical anesthesia.

\section{FLACS}

Femtosecond laser step was applied by the Catalys Precision platform (Johnson \& Johnson Vision) and used for creation of capsulotomy and nucleus fragmentation prior to phacoemulsification of fragmented nucleus parts. Initially, centralization of the Liquid Optics Interface (LOI ${ }^{\mathrm{TM}}$ ) suction cone over the cornea was done, then vacuum was turned on. After confirming good suction together with globe fixation, filling of the suction with balanced saline solution (BSS) was done followed by docking of the patient's eye. An incorporated 3D spectraldomain optical coherence tomography (OCT) measured the dimensions of the anterior chamber and crystalline lens. The surgeon according to each case then modified treatment plan and zones. Finally, the planned treatment was proposed.

For lens pre-fragmentation, we applied sextants with soften fragmentation pattern. We used settings of $350 \mu \mathrm{m}$ grid spacing, $200 \mu \mathrm{m}$ spacing in segmentation-softening pattern, and maximum fragmentation diameter (Figure 1). Segmentation repetition was changed according to nuclear densities as follows: $2-3$ in soft density nuclei, $3-4$ in 


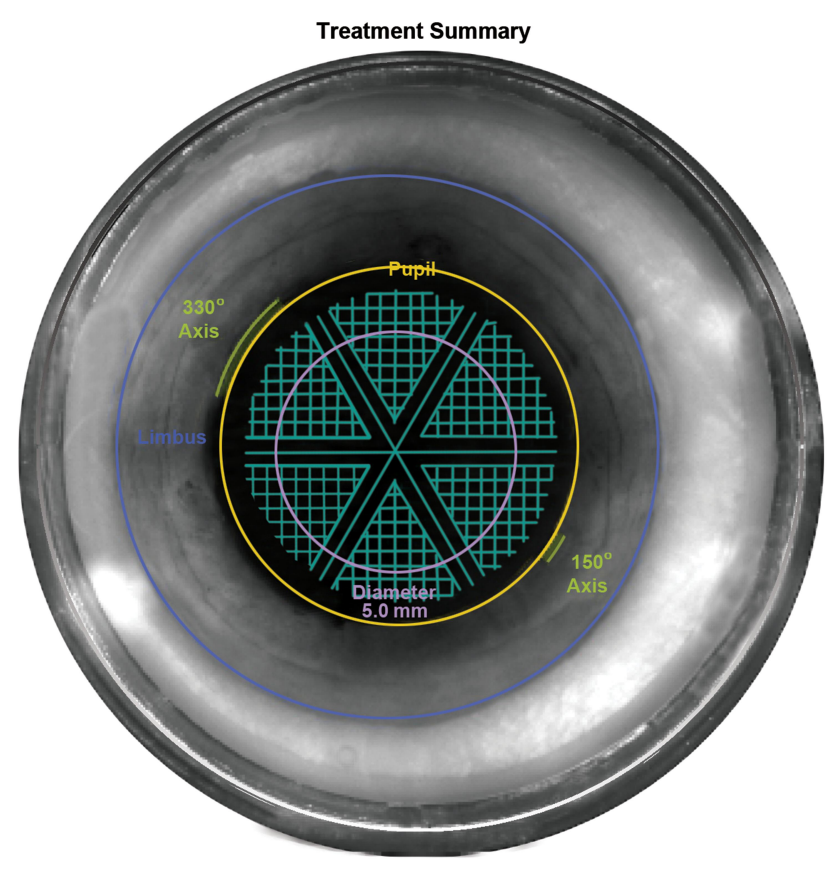

Figure I Lens fragmentation and softening patterns.

medium density nuclei, and 5-6 in hard density nuclei. Other FL parameters were used in its default values; anterior capsule safety margin $500 \mu \mathrm{m}$, posterior capsule safety margin $500 \mu \mathrm{m}$, vertical spot spacing $40 \mu \mathrm{m}$, horizontal spot spacing $10 \mu \mathrm{m}$, anterior pulse energy $10 \mu \mathrm{J}$, and posterior pulse energy $40 \mu \mathrm{J}$.

\section{Phacoemulsification in FLACS and QCP Groups}

Phacoemulsification was performed by the same platform: Signature Pro WhiteStar FX (Johnson \& Johnson Vision) in both FLACS and QCP groups using an ELLIPS FX transversal US phacoemulsification handpiece. The following settings were applied in the two groups: US power of $10-40 \%$ according to the nuclear density, flow rate was set at $28-32 \mathrm{cc} / \mathrm{min}$, vacuum was $350 \mathrm{mmHg}$.

Corneal main incision was done using $2.2 \mathrm{~mm}$ keratome, and secondary incision was done using $1.2 \mathrm{~mm}$ keratome. Filling the anterior chamber was done by cohesive viscoelastic. Capsulorhexis was done manually in the CQCP group by capsulorhexis forceps. While in the FLACS group the FL-created capsulorhexis was detached by capsulorhexis forceps.

Hydrodissection was done as usual. However, it was gentle with a smaller amount of BSS in the FLACS group just to free air bubbles trapped behind the nucleus. Then phacoemulsification of the nucleus was done using a 21
G laminar flow tip by minimal ultrasound energy and high vacuum (350 $\mathrm{mmHg}$ ) in both groups. In the QCP group, vertical quick chopping technique of the nucleus was performed. In the FLACS group, chopping and emulsification of the created sextants was done.

By conclusion of the surgery, we recorded the EFX, which is a value that refers to the total utilized ultrasound time in relation to the amount of used phacoemulsification power. It stands for the effective phacoemulsification time when we use the ELLIPS FX handpiece particularly in Signature Pro platform.

\section{Statistical Analysis}

Statistical data analysis was done using Medcalc Statistics version 18.9.1 (MedCalc Software bvba, Ostend, Belgium). Normality of data was assessed by means of the D'Agostino-Pearson test. The Mann-Whitney test, Kruskal-Wallis test, Conover post-hoc test, and Jonckheere-Terpestra trend test were used to estimate the significance of differences as parametric analyses were not applicable. For all statistical tests, a $P$ value of less than 0.05 was considered as statistically significant.

\section{Results}

This study enrolled 250 eyes of 145 patients (68 males and 77 females), 117 eyes of which were in FLACS group and 133 eyes in QCP group. Subgroup I included eyes with soft nuclear cataracts (NC 1-2) (35 FLACS operated and 42 QCP operated). Subgroup II included eyes with medium nuclear cataracts (NC 3-4) (40 FLACS operated and 47 QCP operated). Subgroup III included eyes with hard nuclear cataracts (NC 5-6) (42 FLACS operated and 44 QCP operated).

Regarding the mean age of the participants, the difference was statistically non-significant either between the FLACS and CQCP groups $(P=0.959)$ or between their underlying subgroups, including subgroup I, II, and III where $P$ values were $0.998,0.542$, and 0.769 , respectively. (Table 1 ).

The surgical parameters documented in both groups including EFX, average US\%, and total US time are illustrated in Table 2 with no statistically significant differences between FLACS and QCP groups regarding these parameters.

The surgical parameters reported in subgroups I, II, and III are summarized in Table 3. The EFX in subgroup I (soft $\mathrm{NC)}$ tended to be lower in the FLACS group in comparison to the QCP group, yet this difference did not reach statistical significance $(P=0.283)$. In subgroup III (hard NC), lower values of EFX were noticed in the FLACS group, but the differences in comparison to the QCP group were not 
Table I Age of the Participants in FLACS and QCP Groups and Subgroups

\begin{tabular}{|l|c|c|c|c|}
\hline \multicolumn{2}{|l|}{ Groups } & N & Age (Mean \pm SD) Yrs & P value \\
\hline \multicolumn{2}{|l|}{ FLACS group } & 117 & $62.5 \pm 8.1$ & \multirow{2}{*}{0.959} \\
\hline \multirow{2}{*}{ QCP group } & 133 & $62.4 \pm 8.4$ & \\
\hline \multirow{2}{*}{ Subgroup I } & FLACS & 35 & $60.2 \pm 11.6$ & \multirow{2}{*}{0.998} \\
\cline { 2 - 4 } & QCP & 42 & $60.4 \pm 10.7$ & \\
\hline \multirow{2}{*}{ Subgroup II } & FLACS & 40 & $60.3 \pm 4.6$ & \multirow{2}{*}{0.542} \\
\cline { 2 - 4 } & QCP & 47 & $64.3 \pm 6.6$ & \\
\hline Subgroup III & FLACS & 42 & $62.8 \pm 6.8$ & \multirow{2}{*}{0.769} \\
\cline { 2 - 4 } & QCP & 44 & $62.8 \pm 7.3$ & \\
\hline
\end{tabular}

Abbreviations: FLACS group, femtosecond laser-assisted cataract surgery group; QCP group, quick chop phacoemulsification group; $n$, number of eyes; SD, standard deviation; yrs, years.

Table 2 Surgical Parameters Documented in FLACS and QCP Groups Including EFX, Average US \% and Total US Time

\begin{tabular}{|l|c|c|c|}
\hline Surgical & $\begin{array}{c}\text { FLACS Group }(\boldsymbol{n}=1 \mathrm{I} \text { 17) } \\
\text { Mean } \pm \text { SD }\end{array}$ & $\begin{array}{c}\text { QCP Group }(\boldsymbol{n}=133) \\
\text { Mean } \pm \text { SD }\end{array}$ & P value \\
\hline EFX (s) & $27.38 \pm 22.15$ & $30.16 \pm 22.59$ & 0.23 \\
Average US \% & $5.9 \pm 3.3$ & $5.8 \pm 3.4$ & 0.45 \\
Total US time (s) & $72 \pm 45.8$ & $66.5 \pm 45$ & 0.44 \\
\hline
\end{tabular}

Abbreviations: FLACS group, femtosecond laser-assisted cataract surgery group; QCP group, quick chop phacoemulsification group; $n$, number of eyes; SD, standard deviation; US, ultrasound; s, seconds.

statistically significant $(P=0.122)$. But for subgroup II, EFX was significantly lower in the FLACS group in comparison to the QCP group $(P<0.0001)$.

\section{Discussion}

Our study compared the EFX used in FLACS to that used in QCP when dealing with soft, hard, and medium density nuclear cataract.

Analysis of the total cohort of the two main groups showed a trend of FLACS to reduce EFX, but this trend did not reach statistical significance. To our knowledge, this could be attributed to the fact that the applied quick phaco chop technique reduces the EFX when compared with other phacoemulsification techniques. $^{23,24}$ This was in concordance with a recent study by Mursch-Edlmayr et al, who found no significant difference between the techniques as regards phacoemulsification energy. ${ }^{13}$ In contrast, a landmark meta-analysis evaluating published studies comparing FLACS and CP, without specifying the used quick phaco chop technique only, showed a significant trend for a difference in EFX in favor of FLACS. ${ }^{17}$

Regarding the used FL segmentation pattern in the FLACS group (Figure 1), we applied nuclear segmentation into sextants. This pattern was expected to help for nucleus division into small segments for easier manipulation and emulsification with less EFX.

In addition, FL fragmentation pattern of the nuclear segments has been proven to be the main factor contributing to the reduction of effective phacoemulsification time in FLACS. ${ }^{25,26}$ Daya et al 2014 concluded that FL fragmentation significantly reduced effective phacoemulsification time compared to $\mathrm{CP}^{27}$

Moreover, the used $350 \mu \mathrm{m}$ grid softening has been demonstrated to lead to a statistically significant lower effective phacoemulsification time than other types of FL fragmentation grids. ${ }^{25}$ Based on our knowledge, the used FL segmentation and fragmentation patterns together with FL softening resulted in ample reduction of phacoemulsification energy for moderate and hard nuclear cataracts. Although we used the same pattern for soft nuclear cataracts, the reduction of EFX was not significant compared to QCP.

Our subgroup analysis showed that this FLACS did not reduce the EFX for all cataract densities. Our study showed non-significant differences regarding EFX between the FLACS group and the QCP group in subgroup I with soft nuclear cataracts $(P=0.283)$. This finding could be explained by the minimal or almost nil

Table 3 The EFX Recorded in the Three Subgroups I, II, and III

\begin{tabular}{|c|c|c|c|c|c|c|c|c|c|}
\hline \multirow[t]{2}{*}{ Parameters } & \multicolumn{3}{|c|}{ Subgroup I (NC I-2) } & \multicolumn{3}{|c|}{ Subgroup II (NC 3-4) } & \multicolumn{3}{|c|}{ Subgroup III (NC 5-6) } \\
\hline & $\begin{array}{c}\text { FLACS } \\
\text { Group } \\
(n=35) \\
M \pm \text { SD }\end{array}$ & $\begin{array}{c}\text { QCP } \\
\text { Group } \\
(n=42) \\
M \pm S D\end{array}$ & $P$ value & $\begin{array}{c}\text { FLACS } \\
\text { Group } \\
(n=40) \\
M \pm \text { SD }\end{array}$ & $\begin{array}{c}\text { QCP } \\
\text { Group } \\
(n=47) \\
M \pm S D\end{array}$ & $P$ value & $\begin{array}{c}\text { FLACS } \\
\text { Group } \\
(n=42) \\
M \pm S D\end{array}$ & $\begin{array}{c}\text { QCP } \\
\text { Group } \\
(n=44) \\
\text { M } \pm \text { SD }\end{array}$ & $P$ value \\
\hline $\operatorname{EFX}(s)$ & $2.37 \pm 1.66$ & $2.74 \pm 1.23$ & 0.283 & $22.37 \pm 6.82$ & $30.34 \pm 6.2$ & $<0.0001$ & $52.97 \pm 10.6$ & $56.14 \pm 9.9$ & 0.122 \\
\hline
\end{tabular}

Abbreviations: FLACS, femtosecond laser-assisted cataract surgery group; QCP, quick chop phacoemulsification group; $n$, number of eyes; M, mean; SD, standard deviation; NC, nuclear cataract. 
US energy needed for emulsification of soft nuclear cataract in QCP. Also, phacoemulsification of such soft nuclear cataract depends more on fluidics rather than phacoemulsification energy. This mostly explains why FLACS was not beneficial over QCP in soft nuclei regarding EFX.

In subgroup III with hard brunescent nuclei, EFX was lower in the FLACS group compared to the QCP one but without a statistically significant difference $(P=0.122)$. We believe that the dense lens opacity precluded femtosecond laser energy required for nuclear fragmentation from reaching the deeper layers of such nuclei. Despite increasing the rate of segmentation repetition up to 6 times, aiming to increase the FL pulse frequency, yet we did not have a complete segmentation in any eye. This can be attributed to two factors. The first factor is that laser energy failed to reach the posterior layers of the nucleus for efficient cracking as we frequently found dense epinucleus posterior sheet, which was not touched by the laser. By default, the Catalys laser precision platform cannot extend laser farther than $500 \mu \mathrm{m}$ off the determined posterior capsule. Hence, a mechanical chopping technique like QCP group was utilized with comparable ultrasound energy. The second factor is that the vertical quick chopping technique employed in the QCP group was effective in the reduction of ultrasound energy by chopping the nucleus into small pieces. ${ }^{23,24}$ This reduction brought down the ultrasound energy to become equivalent to that in the FLACS group. Probably, a significant difference in favor of the FLACS group would have been expected if the divide and conquer technique was applied in this subgroup.

As for subgroup II with moderate hardness cataract, the difference between FLACS and QCP reached statistical significance $(P<0.0001)$. This is because of the capability of FL to perform total nuclear cracking and sufficient softening with subsequent very low or zero US energy for emulsification of the nucleus. This suggests a potentially higher benefit of FLACS over QCP in cataracts with moderately hard nuclei. This agreed with the findings reported by Mayer et al, which demonstrated that FLACS allowed a significant decrease in effective phacoemulsification time correlated with the preoperative lens opacity. ${ }^{28}$

Regarding the unequal number of eyes in the two groups, it is because they were collected from the surgeons' private clinics, and each patient's choice between FLACS and QCP was influenced by the cost factor. The cost difference between the two procedures in our country, Egypt, is about 2000 US dollars in favor of FLACS, which might not be affordable by a lot of patients in our country. Also, as our study is a prospective one, thus from the ethical point of view we must let the patient choose between the two techniques.

\section{Study Limitations}

The $21 \mathrm{G}$ phacoemulsification tip, used in our study, has a narrower $0.5 \mathrm{~mm}$ inner diameter; we presume that 19 $\mathrm{G}$ ones with a wider inner diameter of $0.7 \mathrm{~mm}$ might aid in more reduction in US energy and would have helped to remove pieces of the FL fragmented nucleus by vacuum with less US energy.

Also, we did not compare the effect of reduction of EFX on the surrounding tissues like corneal endothelium, which is under investigation by another study with a larger cohort.

\section{Conclusion}

FLACS significantly reduces EFX during emulsification of medium density nuclear cataracts in comparison to QCP. Regarding very hard and soft nuclear cataracts, FLACS is not superior over QCP in reducing the EFX.

\section{Abbreviations}

EFX, effective phacoemulsification time; $\mathrm{CP}$, conventional phacoemulsification; QCP, quick chop phacoemulsification; FL, femtosecond laser; FLACS, femtosecond laser-assisted cataract surgery; G, gauge; IOL, intraocular lens; LOCS III, Lens Opacities Classification System III; NC, nuclear cataract; PNS, Pentacam Nucleus Staging; US, ultrasound.

\section{Disclosure}

The authors report no conflicts of interest for this work.

\section{References}

1. Nagy ZZ, McAlinden C. Femtosecond laser cataract surgery. Eye Vis (Lond). 2015;2:11. doi:10.1186/s40662-015-0021-7

2. Rivera RP, Hoopes, Jr. PC, Linn SH, Hoopes PC. Comparative analysis of the performance of two different platforms for femtosecond laser-assisted cataract surgery. Clin Ophthalmol. 2016;10:2069-2078. doi:10.2147/OPTH.S115483

3. Yu AY, Lin CX, Wang QM, et al. Safety of femtosecond laser-assisted cataract surgery: assessment of aqueous humour and lens capsule. Acta Ophthalmol. 2016;94:534-540. doi:10.1111/aos.13022

4. Chee SP, Yang Y, Ti SE. Clinical outcomes in the first two years of femtosecond laser- assisted cataract surgery. Am $J$ Ophthalmol. 2015;159:714-719. doi:10.1016/j.ajo.2015.01.016

5. Agarwal A, Jacob S. Current and effective advantages of femto phacoemulsification. Curr Opin Ophthalmol. 2017;28:49-57. doi:10.1097/ICU.0000000000000333

6. Chen X, Yu Y, Song X, et al. Clinical outcomes of femtosecond laser-assisted cataract surgery versus conventional phacoemulsification surgery for hard nuclear cataracts. $J$ Cataract Refract Surg. 2017;43:486-491. doi:10.1016/j.jcrs.2017.01.010

7. Abell RG, Kerr NM, Vote BJ. Toward zero effective phacoemulsification time using femtosecond laser pretreatment. Ophthalmology. 2013;120:942-948. doi:10.1016/j.ophtha.2012.11.045

8. Conrad-Hengerer I, Hengerer FH, Schultz T, Dick HB. Effect of femtosecond laser fragmentation on effective phacoemulsification time in cataract surgery. $J$ Refract Surg. 2012;28:879-883. doi:10.3928/1081597X-20121116-02 
9. Mastropasqua L, Mattei PA, Toto L, et al. All laser cataract surgery compared to femtosecond laser phacoemulsification surgery: corneal trauma. Int Ophthalmol. 2017;37:475-482. doi:10.1007/s10792-0160283-7

10. Abell RG, Kerr NM, Howie AR, et al. Effect of femtosecond laser-assisted cataract surgery on the corneal endothelium. $J$ Cataract Refract Surg. 2014;40:1777-1783. doi:10.1016/j. jcrs.2014.05.031

11. Scott WJ, Tauber S, Gessler JA, et al. Comparison of vitreous loss rates between manual phacoemulsification and femtosecond laser-assisted cataract surgery. $J$ Cataract Refract Surg. 2016;42:1003-1008. doi:10.1016/j.jcrs.2016.04.027

12. Taravella MJ, Meghpara B, Frank G, et al. Femtosecond laserassisted cataract surgery in complex cases. J Cataract Refract Surg. 2016;42:813-816. doi:10.1016/j.jcrs.2016.02.049

13. Mursch-Edlmayr AS, Bolz M, Luft N, et al. Intraindividual comparison between femtosecond laser-assisted and conventional cataract surgery. J Cataract Refract Surg. 2017;43:215-222. doi:10.1016/j. jcrs.2016.11.046

14. Manning S, Barry P, Henry Y, et al. Femtosecond laser-assisted cataract surgery versus standard phacoemulsification cataract surgery: study from the European Registry of Quality Outcomes for cataract and refractive surgery. J Cataract Refract Surg. 2016;42:1779-1790. doi:10.1016/j.jcrs.2016.10.013

15. Ewe SY, Abell RG, Oakley CL, et al. A comparative cohort study of visual outcomes in femtosecond laser- assisted versus phacoemulsification cataract surgery. Ophthalmology. 2016;123:178-182. doi:10.1016/j.ophtha.2015.09.026

16. Abell RG, Darian-Smith E, Kan JB, et al. Femtosecond laser- assisted cataract surgery versus standard phacoemulsification cataract surgery: outcomes and safety in more than 4000 cases at a single center. $J$ Cataract Refract Surg. 2015;41:47-52. doi:10.1016/j. jcrs.2014.06.025

17. Popovic M, Campos-Möller X, Schlenker MB, Ahmed II. Efficacy and safety of femtosecond laser-assisted cataract surgery compared with manual cataract surgery: a meta-analysis of 14567 eyes. Ophthalmology. 2016;123:2113-2126. doi:10.1016/j. ophtha.2016.07.005

18. Berk TA, Schlenker MB, Campos-Mo“ller X, et al. Visual and refractive outcomes in manual versus femtosecond laser-assisted cataract surgery: a single-center retrospective cohort analysis of 1838 eyes. Ophthalmology. 2018;125(8):1172-1180. doi:10.1016/j.ophtha. 2018.01.028
19. Conrad-Hengerer I, Al Sheikh M, Hengerer FH, et al. Comparison of visual recovery and refractive stability between femtosecond laser-assisted cataract surgery and standard phacoemulsification: six-month follow-up. $J$ Cataract Refract Surg. 2015;41 (7):1356-1364. doi:10.1016/j.jcrs.2014.10.044

20. Chylack LT, Wolfe JK, Singer DM, et al. The lens opacities classification system III, the longitudinal study of cataract study group. Arch Ophthalmol. 1993;111(6):831-836. doi:10.1001/archopht.1993.01090060119035

21. Hida WT, Tzelikis PF, Vilar C, et al. Outcome study between femtosecond laser-assisted cataract surgery and conventional phacoemulsification surgery using an active fluidics system. Clin Ophthalmol. 2017;11:1735-1739. doi:10.2147/OPTH.S136136

22. Aly MG, Shams A, Fouad YA, Hamza I. Effect of lens thickness and nuclear density on the amount of laser fragmentation energy delivered during femtosecond laser-assisted cataract surgery. J Cataract Refract Surg. 2019;45(4):485-489. doi:10.1016/j.jcrs.2018.11.014

23. Park J, Yum HR, Kim MS, Harrison AR, Kim EC. Comparison of phaco-chop, divide-and-conquer, and stop-and-chop phaco techniques in microincision coaxial cataract surgery. $J$ Cataract Refract Surg. 2013;39(10):1463-1469. doi:10.1016/j.jcrs.2013.04.033

24. Park JH, Lee SM, Kwon JW, et al. Ultrasound energy in phacoemulsification: a comparative analysis of phaco-chop and stop-and-chop techniques according to the degree of nuclear density. Ophthalmic Surg Lasers Imaging. 2010;41(2):236-241. doi:10.3928/1542887720100303-13

25. Conrad-Hengerer I, Hengerer FH, Schultz T, Dick HB. Effect of femtosecond laser fragmentation of the nucleus with different softening grid sizes on phaco time in cataract surgery. $J$ Cataract Refract Surg. 2012;38:1888-1894. doi:10.1016/j.jcrs.2012.07.023

26. Kim DH, Wee WR, Lee JH, Kim MK. The comparison between torsional and conventional mode phacoemulsification in moderate and hard cataracts. Korean J Ophthalmol. 2010;24:336-340. doi:10.3341/kjo.2010.24.6.336

27. Daya SM, Nanavaty MA, Espinosa-Lagana MM. Translenticular hydrodissection, lens fragmentation, and influence on ultrasound power in femtosecond laser- effective assisted cataract surgery and refractive lens exchange. J Cataract Refract Surg. 2014;40:37-43. doi:10.1016/j.jcrs.2013.07.040

28. Mayer WJ, Klaproth OK, Hengerer FH, Kohnen T. Impact of crystalline lens opacification on effective phacoemulsification time in femtosecond laser-assisted cataract surgery. Am J Ophthalmol. 2014;157:426-432. doi:10.1016/j.ajo.2013.09.017
Clinical Ophthalmology

\section{Publish your work in this journal}

Clinical Ophthalmology is an international, peer-reviewed journal covering all subspecialties within ophthalmology. Key topics include: Optometry; Visual science; Pharmacology and drug therapy in eye diseases; Basic Sciences; Primary and Secondary eye care; Patient Safety and Quality of Care Improvements. This journal is indexed on PubMed
Central and CAS, and is the official journal of The Society of Clinical Ophthalmology (SCO). The manuscript management system is completely online and includes a very quick and fair peer-review system, which is all easy to use. Visit http://www.dovepress.com/ testimonials.php to read real quotes from published authors.

\section{Dovepress}

